\title{
Uso terapêutico da radiofrequência pulsátil no gânglio dorsal da raiz de L2 na lombalgia discogênica
} Pulsed radiofrequency on $L 2$ dorsal root ganglion as a therapeutic method for lumbar discogenic pain Uso terapéutico de la radiofrecuencia pulsátil en el ganglio dorsal de la raíz de L2 en la lumbalgia discogénica

\author{
Fabrício Dias Assis ${ }^{1}$ \\ Charles Amaral' \\ Carlos Tucci' \\ Sílvia Maria Bordignon da Costa ${ }^{3}$
}

\section{RESUMO}

Objetivo: Avaliar a eficácia da radiofrequência pulsátil sobre o gânglio da raiz dorsal de L2 no tratamento dos pacientes com lombalgia discogênica. Métodos: Realizou-se análise retrospectiva de 50 pacientes portadores de lombalgia crônica discogênica atendidos no período de janeiro de 2004 a julho de 2007. O processo diagnóstico foi constituído por exame físico, ressonância magnética e bloqueio diagnóstico do gânglio da raiz dorsal de L2. Todos os pacientes foram submetidos à radiofrequência pulsátil no gânglio da raiz dorsal de L2 e acompanhados por, no mínimo, 12 meses. A intensidade de dor foi medida pela escala visual analógica (EVA) de dor. Resultados: A análise estatística mostrou melhora significativa da intensidade de dor $(\mathrm{p}<0,001)$ após 12 meses de seguimento. $\mathrm{O}$ uso da radiofrequência pulsátil no gânglio da raiz dorsal de L2 mostrou-se um método alternativo inespecífico eficaz

\section{ABSTRACT}

Objective: to evaluate the effectiveness of pulsate radio-frequency on L2 dorsal root ganglion for chronic discogenic low back pain. Of L2 in the treatment of the patient with discogenic low back pain. Methods: Between January 2004 and July 2007, 50 patients with diagnosis of low back discogenic pain were retrospectively assessed based on physical examination, magnetic resonance imaging findings and selective L2 dorsal root ganglion diagnostic block, and then submitted to pulsed radiofrequency on L2 dorsal root ganglion, and evaluated after a minimum follow-up of 12 months. Pain was evaluated by the visual analog scale. Results: Statistical analysis showed significant improvement of pain intensity $(p<0.001)$ after a minimum 12-month follow-up period. Conclusion: Pulsed radiofrequency on L2 dorsal root ganglion is an effective, non-specific therapeutic method for low back discogenic pain,
\end{abstract}

\section{RESUMEN}

Objetivo: evaluar la eficacia de la radiofrecuencia pulsátil sobre el ganglio de la raíz dorsal de L2 en el tratamiento de los pacientes con lumbalgia discogénica. Métodos: fue realizado un análisis retrospectivo de 50 pacientes portadores de lumbalgia crónica discogénica, atendidos en el periodo de Enero de 2004 a Julio de 2007. El proceso diagnóstico constó de un examen fisico, resonancia magnética y bloqueo diagnóstico del ganglio de la raíz dorsal de L2. Todos los pacientes fueron sometidos a la radiofrecuencia pulsátil en el ganglio de la raiz dorsal de L2 y seguidos por 12 meses, como mínimo. La intensidad del dolor fue medida por la escala visual analógica del dolor. Resultados: el análisis estadístico mostró mejoría significativa de la intensidad del dolor $(p<0.001)$ después de 12 meses de seguimiento. Conclusión: El uso de la radiofrecuencia pulsátil en el ganglio de la raíz dorsal de L2 mostró ser un método inespecífico, eficaz y alternativo

\footnotetext{
Trabalho realizado na Pontifícia Universidade Católica de Campinas - PUC-CAMPINAS - Campinas (SP), Brasil; Ambulatório de Dor Crônica da Clínica Sindolor Campinas (SP), Brasil.

'Terapeuta de dor da Clínica Sindolor - Campinas (SP), Brasil.

${ }^{2}$ Cirurgião da coluna, chefe do Grupo de Coluna da Pontifícia Universidade Católica de Campinas - PUC- CAMPINAS - Campinas (SP), Brasil.

${ }^{3}$ Enfermeira da Clínica Sindolor - Campinas (SP), Brasil. 
às cirurgias convencionais, apesar do curto seguimento desta série. despite the short follow-up period of this series. a las cirugías convencionales, a pesar del corto seguimiento de hecho en esta serie.

DESCRIPTORES: Dolor de la región lumbar/terapia; Dolor de la región lumbar/ diagnóstico; Imagen por resonancia magnética; Dimensión del dolor; Ondas radioeléctricas/uso terapéutico

\section{INTRODUÇÃO}

Dor lombar crônica é definida quando permanece por pelo menos três meses. Essa entidade é de alta prevalência na população mundial, gerando custos sociais e econômicos de grandes proporções.

A dor de origem discal (ou discogênica) está entre as causas mais frequentes de lombalgia crônica, juntamento com a artrose facetária, a sacroileíte e as radiculopatias inespecíficas.

A comprovação diagnóstica da dor lombar discogênica (DLD) depende do estudo fisiológico da dor ${ }^{1,2}$, dos exames de imagens tradicionais (radiografia simples e ressonância magnética), discografia e, mais recentemente, dos bloqueios diagnósticos (BD). Seu tratamento permanece, no entanto, controverso ${ }^{3-5}$.

O presente estudo tem como objetivo comprovar o efeito diagnóstico do bloqueio do gânglio da raiz dorsal de L2 e a eficácia da aplicação da radiofrequência pulsátil sobre essa raiz como opção terapêutica para a lombalgia discogênica.

\section{MÉTODOS}

Realizou-se análise retrospectiva de pacientes atendidos no período de Janeiro de 2004 a Julho de 2007 no ambulatório de dor crônica da Clínica Sindolor. A pesquisa incluiu 50 pacientes (18 indivíduos do sexo masculino e 32 do sexo feminino) com média de idade de 56,67 anos (variação de 18 a 88 anos), portadores de dor lombar discogênica com intensidade de dor média de 7,71 (variando de 3 a 10).

O diagnóstico foi baseado nos exames físico e de ressonância magnética e no bloqueio anestésico diagnóstico. A intensidade da dor foi medida pela escala visual analógica (EVA).

A dor discogênica tem caráter mecânico e, em geral, é referida no dermátomo de L2; os pacientes podem referir dor quando sentados por longos períodos, à flexão associada à rotação e ao final da atividade.

As imagens de ressonância magnética foram obtidas em um aparelho de 1,5 Tesla (Siemens ${ }^{\circledR}$, Erlang, Alemanha), ponderadas em T1 e T2 nos cortes axial e sagital e classificadas conforme Pfirmann ${ }^{6}$.
O gânglio da raiz dorsal (GRD) foi bloqueado com anestésico local $(0,5 \mathrm{~mL}$ de Lidocaína a $2 \%$ sem vasoconstritor), sob fluoroscopia (Figura 1). O exame foi considerado positivo quando a percepção dolorosa diminuiu pelo menos 50\%, com base na EVA obtida após o procedimento.
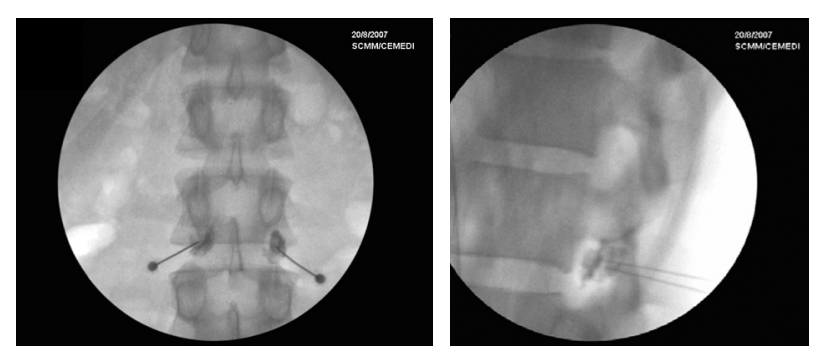

Figura 1

Localização do gânglio da raiz dorsal de L2

Nos pacientes confirmados como portadores de dor lombar discogênica, a radiofrequência pulsátil (Baylis Medical $\mathrm{PMG}^{\circledR}$ - 230 [-TD], Montreal, Canadá) a 45V, limitada a $42{ }^{\circ} \mathrm{C}$ com dois ciclos de $20 \mathrm{~ms} / \mathrm{s}$ e impedância menor que $400 \mathrm{ohms}$, foram aplicadas no gânglio da raiz dorsal da raiz de L2 sob fluoroscopia por 180 segundos, em num período que variou de uma semana a três meses do bloqueio diagnóstico.

Todos os 50 pacientes foram acompanhados por pelo menos um ano e avaliados pela EVA no $3^{\circ}, 6^{\circ}$ e $12^{\circ}$ meses pós-procedimento.

A análise exploratória estatística foi feita através do software SAS (Statistical Analysis System, SAS Institute ${ }^{\circledR}$, Cary, NC, Estados Unidos da América), aplicando-se também o teste $t$ de Student, com intervalo de confiança de $95 \%$

\section{RESULTADOS}

O tempo de seguimento mínimo de 12 meses foi o critério de inclusão. Todos os pacientes foram submetidos à avaliação de dor apenas pela EVA.

A intensidade de dor pela EVA inicial média foi de 7,71 pré-bloqueio e de 3,62, 3,15 e 2,83, respectivamente após 3, 6 e 12 meses da aplicação da radiofrequência pulsátil. 
Não foram observadas complicações relacionadas ao bloqueio diagnóstico ou à aplicação da radiofrequência.

A partir do teste estatístico ( $t$ de Student), notou-se diferença significativa nos resultados para idade $(\mathrm{p}=0,0006)$ e intensidade de dor $(p<0,0001)$. Não houve diferença significativa para sexo $(\mathrm{p}=0,95)$.

\section{DISCUSSÃO}

A dor lombar é a causa mais frequente de incapacidade permanente em adultos na faixa dos 45 anos de idade e a segunda causa mais comum de procura por atendimento médico. Oitenta por cento $(80 \%)$ dos adultos experimentarão pelo menos um episódio de dor lombar durante a vida. Destes, $60 \%$ apresentarão recorrência.

Mesmo assim, a grande maioria das crises de lombalgia aguda evolui para a remissão completa. Por outro lado, as queixas que ultrapassam três meses de duração tendem a perdurar ainda mais, agravadas pelos diagnósticos imprecisos e consequentes tratamentos inadequados.

A causa da lombalgia crônica, no entanto, é imprecisa e controversa. Apenas $15 \%$ dos diagnósticos anatômicos são possíveis quando associados a exame físico, radiografia simples, tomografia computadorizada, ressonância magnética e eletroneuromiografia. Os demais $85 \%$ são tratados como dor músculo-ligamentar de fundo psicológico ou fibromialgia.

A DLD afeta indivíduos após a terceira década de vida, principalmente entre a quarta e a quinta décadas. Sua etiologia está relacionada à desidratação e degeneração dos discos intervertebrais, em especial nos segmentos mais caudais (L4/L5 e L5/S1). As forças de rotação e flexão incidentes nesses níveis geram fissuras no ânulo fibroso, desencadeando um processo irreversível de migração centrípeta de vasos e nervos, a partir do ânulo externo, simultâneo à desidratação fisiológica do núcleo pulposo, tornando o disco intervertebral doloroso.

A dor de origem discal é bem difundida como causa de dor lombar crônica ${ }^{7,8}$. A discografia é o teste padrão de diagnóstico específico para a dor discogênica; quando bem realizada e bem interpretada, é específica e não produz falso-negativo $0^{9,10}$.

O bloqueio diagnóstico do gânglio da raiz dorsal de L2 surgiu como alternativa à discografia baseado nos estudos anatômicos de Nakamura et al..$^{1,2}$, que determinaram a transmissão de dor pelas fibras simpáticas aferentes que emergem no gânglio da raiz dorsal de L2, confirmando as conclusões de estudos anteriores ${ }^{11,12}$.

O bloqueio diagnóstico do gânglio da raiz dorsal de L2 tem sido utilizado pelos autores como ferramenta diagnóstica de dor discogênica inespecífica.

Quando confirmada, a dor discogênica lombar é passível de tratamento com aplicação de radiofrequência pulsátil no gânglio da raiz dorsal de L2, modulando a transmissão aferente de estímulos nociceptivos ${ }^{13}$.

\section{CONCLUSÃO}

A radiofrequência pulsátil sobre o gânglio da raiz dorsal de L2 é uma opção segura e eficaz para o tratamento da dor lombar discogênica, com altos índices de melhora de dor, como comprovou esta série. Sua principal limitação é a inespecificidade, tratando qualquer disco degenerado abaixo de L2, contrariamente às alternativas cirúrgicas convencionais. O presente estudo é limitado pelo curto seguimento dos indivíduos incluídos e pela falta de uma ferramenta de avaliação de qualidade de vida. Entretanto, esta é a primeira série que avalia a radiofrequência pulsátil no gânglio da raiz dorsal e L2 com seguimento mínimo de 12 meses.

\section{REFERÊNCIAS}

1. Nakamura S, Takahashi K, Takahashi Y, Morinaga T, Shimada Y, Moriya H. Origin of nerves supplying the posterior portion of lumbar intervertebral discs in rats. Spine. 1996;21(8):917-24.

2. Nakamura SI, Takahashi K, Takahashi Y, Yamagata M, Moriya H. The afferent pathways of discogenic lowback pain. Evaluation of L2 spinal nerve infiltration. J Bone Joint Surg Br. 1996;78(4):606-12.
3. Zigler J, Delamarter R, Spivak JM, Linovitz RJ, Danielson GO 3rd, Haider TT, et al. Results of the prospective, randomized, multicenter Food and Drug Administration investigational device exemption study of the ProDisc-L total disc replacement versus circumferential fusion for the treatment of 1-level degenerative disc disease. Spine. 2007;32(11):1155-62; discussion 1163.

4. Mirza SK, Deyo RA. Systematic review of randomized trials comparing lumbar fusion surgery to nonoperative care for treatment of chronic back pain. Spine. 2007;32(7):816-23. Comment in: J Bone Joint Surg Am. 2007;89(11):2558.
5. Yakovlev A, Tamimi MA, Liang $\mathrm{H}$, Eristavi M. Outcomes of percutaneous disc decompression utilizing nucleoplasty for the treatment of chronic discogenic pain. Pain Physician. 2007;10(2):319-28.

6. Pfirrmann CW, Metzdorf A, Zanetti M, Hodler J, Boos N. Magnetic resonance classification of lumbar intervertebral disc degeneration. Spine. 2001;26(17):1873-8.

7. Peng B, Wu W, Hou S, Li P, Zhang $\mathrm{C}$, Yang Y. The pathogenesis of discogenic low back pain. J Bone Joint Surg Br. 2005;87(1):62-7.

8. Peng B, Hao J, Hou S, Wu W, Jiang D, $\mathrm{Fu}$ X, Yang Y. Possible pathogenesis of painful intervertebral disc degeneration. Spine. 2006;31(5):560-6. 
9. Walsh TR, Weinstein JN, Spratt KF, Lehmann TR, Aprill C, Sayre H. Lumbar discography in normal subjects. A controlled, prospective study. J Bone Joint Surg Am. 1990;72(7):1081-8.

10.Carragee EJ, Paragioudakis SJ, Khurana S. 2000 Volvo Award winner in clinical studies: Lumbar highintensity zone and discography in subjects without low back problems. Spine. 2000;25(23):2987-92.

11. Wiberg G. Back pain in relation to the nerve supply of the intervertebral disc. Acta Orthop Scand. 1949;19(2):21121 , illust.
12.Bogduk N, Tynan W, Wilson AS. The nerve supply to the human lumbar intervertebral discs. J Anat. 1981;132(Pt 1):39-56.

13. Cahana A, Vutskits L, Muller D. Acute differential modulation of synaptic transmission and cell survival during exposure to pulsed and continuous radiofrequency energy. J Pain. 2003;4(4):197-202.

\section{Correspondência}

Carlos Tucci

Rua Maria Monteiro, 968

CEP 13025-151 - Campinas (SP), Brasil

E-mail:carlos@tucci.med.br 